Access blood flow rate 177

- selection 338

- survival 562

Acidosis 550

Acute renal failure 154

Advanced glycation end products 531

- oxidation protein products 531

Anemia 349, 357

Arteriovenous bridge grafts 338

- fistulas 338, 556

Autologous fistula 562

Autonomic dysfunction 150

Biocompatibility 161

Blood flow 376

- pressure 370

Bone marrow 174

Carbonyl stress 531

CD34 antigen 174

Cell adhesion molecules 174

Central catheter 182

Complications of dialysis 150

Congestive heart failure 342

Continuous ambulatory peritoneal dialysis 537

- hemodiafiltration 342

Critical care 342

Cytokines 154

Detoxification 380

Dialysis 568

- adequacy 525

- hypotension 150

- membrane 325

Dialysis-related amyloidosis 161

Dietary intervention 139

Drugs, intoxication 380

eKT/V 550

Elderly, vascular access 562
End-stage renal disease 167, 349

EPO resistance 349

Filtration coefficient 568

Folic acid 334

\section{Grafts 562}

$\mathrm{H}(\mathrm{a})$ emodiafiltration 161,349

$\mathrm{H}(\mathrm{a})$ emodialysis $145,154,177,182,334$, $364,376,525,531,537,543,550,556$, 562

- duration 525

Hematocrit 376, 568

Hemodynamics 342, 364, 556

Hemoperfusion 380

Heparin 145

High-flux (hemo)dialysis 161,325

High-volume substitution fluid 357

Homocysteine 334

Hypotension 145, 364, 568

Immunosuppression 139

Interleukin-10 543

Intimal hyperplasia 556

Intoxication 380

Iron utilization 349

8-Iso-prostaglandin $\mathrm{PGF}_{2 \alpha} 537$

\section{$\mathrm{Kt} / \mathrm{V} 154$}

Lipid peroxidation 537

Long nocturnal hemodialysis sessions 525

Metabolism 139

$\beta_{2}$-Microglobulin 161

Middle molecular weight molecules 380

Monitoring 364

Nitric oxide 145

Nutritional state 139
On-line hemodiafiltration 357

Oxidative stress 531

Parnaparin 145

Peripheral blood mononuclear cells 543

Physical activity 139

Plasma exchange 167

Polysulfone 325

Profiling 364

Protein catabolic rate 550

Recirculation 182

Renal transplantation 139

Serotonin reuptake inhibitors 150

Sertraline hydrochloride 150

Sodium 364

Sorbents 380

Sunlight exposure 370

Survival 167

Tissue oxygen metabolism 342

Tumor necrosis factor- $\alpha 543$

Ultrafiltration 364

Ultrasonography 177

Umbilical cord 174

Urea kinetic modeling 154

- reduction ratio 550

Vascular access 177, 338, 376

Venous cuff 556

Vitamin $B_{12} 334$

- $\mathrm{D}_{3} 370$

VLA-2 174

VLA-5 174

Wegener granulomatosis 167

\title{
KARGER
}

(C) 2002 S. Karger AG, Basel

Fax +4161306 1234

E-Mail karger@karger.ch

www.karger.com 